\title{
Issues in modern bone histomorphometry ${ }^{\star}$
}

\author{
R.R. Recker ${ }^{a}{ }^{\star}$, D.B. Kimmel ${ }^{\mathrm{a}}$, D. Dempster ${ }^{\mathrm{b}}$, R.S. Weinstein ${ }^{\mathrm{c}}$, T.J. Wronski ${ }^{\mathrm{d}}$, and D.B. Burre,f \\ aOsteoporosis Research Center, Creighton University, 601 N 30th St Omaha, NE, 68131, USA \\ ${ }^{\mathrm{b}}$ Regional Bone Center, Helen Hayes Hospital, Route 9W, West Haverstraw, NY, 10993, USA \\ ${ }^{\circ}$ Center for Osteoporosis and Metabolic Bone Diseases, Dept. of Medicine, Central Arkansas \\ Veterans Health Care System and University of Arkansas for Medical Sciences, 4301 West \\ Markham St.- slot 587, Little Rock AR, 72205, USA \\ dDept. of Physiological Sciences, University of Florida, P.O. Box 100144, JHMHC, Gainesville, \\ FL, 32610, USA \\ eDept. of Anatomy and Cell Biology, Indiana University School of Medicine, 635 Barnhill Dr., MS \\ 5035, Indianapolis, IN, 46202, USA \\ fDept. of Biomedical Engineering, Indiana University-Purdue University Indiana (IUPUI), USA
}

\section{Abstract}

This review reports on proceedings of a bone histomorphometry session conducted at the Fortieth International IBMS Sun Valley Skeletal Tissue Biology Workshop held on August 1, 2010. The session was prompted by recent technical problems encountered in conducting histomorphometry on bone biopsies from humans and animals treated with anti-remodeling agents such as bisphosphonates and RANKL antibodies. These agents reduce remodeling substantially, and thus cause problems in calculating bone remodeling dynamics using in vivo fluorochrome labeling. The tissue specimens often contain few or no fluorochrome labels, and thus create statistical and other problems in analyzing variables such as mineral apposition rates, mineralizing surface and bone formation rates. The conference attendees discussed these problems and their resolutions, and the proceedings reported here summarize their discussions and recommendations.

\section{Keywords}

Histomorphometry; Bone remodeling; Over-suppression

\section{Introduction}

The understanding of bone physiology accelerated in the 1950s with the development of plastic-embedding technology for microscopic examination of undemineralized sections of bone tissue. Prior to then, bone histology required removal of its principal component, the mineral. This new technology was refined in several labs, in particular, the Radiobiology Lab at the University of Utah in studies of the skeletal effects of plutonium and radium, alpha radiation-emitting, bone-seeking metals. A benchmark occurred in 1960 with a publication by Frost [1] entitled, "Measurement of Bone Formation in a 57 Year-old Man by

\footnotetext{
Presented at the Fortieth International IBMS Sun Valley Skeletal Tissue Biology Workshop; August 1, 2010.

(C) 2011 Elsevier Inc. All rights reserved.

*Corresponding author at: 601 N 30th St, \#5766, Omaha, NE, 68131, USA. Fax: +1 402280 5034. rrecker@ creighton.edu (R.R. Recker).
} 
Means of Tetracyclines." This opened the door for study of the complete composite of human bone, including both mineral and organic matrix. In 1983, a text was produced [2] which summarized the methodology and stereological theories behind the histomorphometric calculations. Another benchmark occurred in 1987 when the nomenclature, symbols and units for bone histomorphometry were standardized [3]. As a consequence, the use of in-life dual fluorochrome labels in humans and animals together with the application of bone histomorphometric techniques has become a standard method for studying the tissue level activities of bone modeling and remodeling. Starting in the late 1960s, Professor Webster Jee began the Sun Valley Workshops on musculoskeletal biology that continue to the present. In August 2010, the International Bone and Mineral Society, along with the National Institutes of Health, sponsored the 40th Annual IBMS/Sun Valley Workshop on musculoskeletal biology. At that meeting a half-day session was devoted to discussing issues in modern histomorphometry, 50 years after its introduction. The authors participated in the presentations and subsequent discussion.

\section{Change in landscape}

The success of potent anti-remodeling agents in reducing fracture risk in osteoporotic patients in recent years has presented new real world problems for bone histomorphometry, as it is applied to explain their tissue level mechanisms. Bone biopsies taken from patients, or specimens taken from animals treated with these agents, may have few or no fluorochrome labels, creating difficulty in the interpretation of the dynamic histomorphometric data and evaluation of patients' or animals' bone remodeling status. This experience has generated a number of important questions that have been debated in publications and scientific meetings over the past decade. Other issues involve use of proper referents for histomorphometric measurements, clarification of the utility of certain bone histomorphometric measurements and endpoints, sample size requirements for valid measurements, quantification of rare events, and the variables that should be reported to describe best the status of bone remodeling. The August 2010 Symposium sought to summarize the following questions and provide recommendations on how they might be answered:

1. How should one express and interpret mineralizing surface (MS/BS) and bone formation rate (BFR/BS) in the absence or paucity of double and/or single label in the standard sampling area?

2. How should mineral apposition rate (MAR) be expressed in the absence or paucity of double label in the standard sampling area? Should MAR be imputed (i.e., assigned a value)? If so, when?

3. Is an "extended label search" worth doing in the absence of label in the standard sampling area? (The definition of standard sampling area is $2-4$ sections, $\sim 100 \mu$ apart, from the middle one third of a human transiliac biopsy specimen, or embedded animal specimen, sufficient to provide the minimum area for analysis as described later.) What should be the purpose of such a search? If label is found during the search, how should it be expressed?

4. Should MAR be measured and reported if found only on an extended label search? If so, in what manner?

5. What is the minimum and practical maximum number of double label width measurements required to achieve a reliable estimate of MAR? How should one handle situations with insufficient double label to achieve a reliable estimate of MAR? 
6. What is the minimum number of measured sections, area, and perimeter to provide an adequate sample?

7. What is activation frequency? How should one interpret activation frequency? What assumptions are required? Is it a valid expression of remodeling rate?

8. Can one measure "erosion depth" reliably? Is it valuable to do so?

9. Is the "obliquity correction" required for width measurements?

10. Should one examine cortical bone in human transilial biopsies? If so, what variables should be measured?

11. What is the confidence in extrapolations from a transiliac biopsy $(\sim 1 / 14,000$ of the skeleton) to the entire skeleton?

12. Can one define the term "bone anabolic agent" solely in histomorphometric terms? If not, what additional data are needed?

13. What is the definition of "over-suppression" of remodeling? Does "oversuppression of remodeling" exist in a practical sense? Do biochemical markers of bone formation and resorption assist in defining "over-suppression?"

\section{Issues of measurement in low remodeling states}

In bone biopsies that show few or no tetracycline labels after a standard in-life labeling protocol [4,5], a number of related questions emerge pertaining to handling the data. These are particularly important in human clinical studies that use iliac histomorphometry to understand a disease condition or the tissue level mechanisms by which a drug is acting by analyzing and comparing data from groups of subjects. The same questions apply to the evaluation of bone in animals treated with these types of agents in pharmacology studies.

\section{Forming surface}

In vivo fluorochrome labeling to mark bone formation surface is done by the administration of two time-separated (by 14-17 days in humans, 10-14 days in large animals, or 2-7 days in small animals) sequences of label shortly before obtaining the bone specimen. Thus, in fluorochrome-labeled trabecular bone in human transilial biopsies, the overall forming surface is defined as including both singly and doubly labeled surfaces. Because of the "label escape" phenomenon [6,7], the most accurate measure of forming surface, mineralizing surface (MS/BS), includes the combination of doubly labeled perimeter plus half the singly labeled perimeter as a fraction (or percent) of the total trabecular perimeter, i.e., $\mathrm{MS} / \mathrm{BS}=100 *(\mathrm{dL} . \mathrm{Pm}+(0.5 * \mathrm{sL} . \mathrm{Pm})) / \mathrm{B} . \mathrm{Pm}$ (Table 2$)$. MS/BS is the best expression of the extent of surface remodeling in any specimen. This important variable is then used to calculate a number of derived variables that express bone remodeling rates.

Three types of problems arise when labels of either type (double or single) are scarce or absent in the standard analysis region, something that has been repeatedly encountered during the study of anti-remodeling agents:

1. By examining the MS/BS formula, it can be appreciated that specimens that have neither double nor single label in the standard analysis region have MS/BS of zero, leading directly to an implied bone formation rate (BFR/BS) of zero (Table 2). Specimens that have double label, but lack single label present no problem.

2. Other specimens that have only single label in the standard analysis region will have non-zero MS/BS, but a non-calculable bone formation rate because mineral apposition rate (MAR) cannot be calculated due to the absence of double labels. Without double labels to measure, zero appears in the denominator $(\mathrm{N}$, number of 
measurements taken) of the MAR formula, and in the denominator of the derived variables in which it appears.

3. Still other specimens have so little double label in the standard analysis region that they provide an insufficient sample for reliable assessment of MAR. These have both non-zero MS/BS and nonzero bone formation rates, but lack reliability due to an inadequate number of double labels available to sample for MAR.

In analyzing central trends and treatment effects in groups of patients, experience has shown that the method of handling specimens with no or insufficient label in the standard analysis region can produce significant bias in the analyses. Clearly, if specimens with no label in the standard analysis region are simply treated as "missing data" and excluded from their groups' analyses, the result is an unacceptable bias toward overestimating remodeling rates in the group. If specimens with insufficient label in the standard analysis region (see sections on Change in landscape and Extended label search) are given the same weight as those with adequate amounts of label, the result is an unacceptable reliance on poor data. Our recommendations regarding the calculation of variables expressing bone formation rate when double label is absent in the standard analysis region follow:

For the simpler variable, MS/BS, we recommend that the actual value always be reported. Reporting "zero" for MS/BS when no label of any type is found is proper because it accurately reflects the observations in the specimen. Reporting the actual value for MS/BS when only single label is found, is proper because it again accurately reflects the data of the specimen. Reporting the actual value for MS/BS when only one or two double labels are found is proper because it not only reflects the specimen data accurately, but also has been obtained on an adequate sample of bone perimeter.

For MAR, we recommend that the actual value be reported for a patient when it is non-zero and sampling has been adequate (see subsequent discussion). We recommend that when no label of any type is found in the standard analysis region, MAR should be reported as "missing data" for that patient. It properly represents what was observed. There was no double label upon which to base the calculation of MAR. We recommend that when only single label is found in the standard analysis region, MAR be reported as "missing data" for that patient. When only single label is found, it is also permissible to impute (assign) a value for MAR (see Section Issues of measurement in low remodeling states). When an imputed value for MAR is used calculations should be reported in both ways - one with MAR as "missing data" and a second set using the imputed value of MAR. We recommend that when insufficient double label perimeter is present for measuring MAR (see Section Extended label search), the actual value for MAR is reported, but flagged as based on insufficient double label evaluation and noted on the patient/animal report.

For the more complex variable, BFR/BS (Table 2), we recommend that the actual value be reported for a patient when it is non-zero and sampling has been adequate for MAR (see Section Extended label search). We recommend that when no label of any type is found in the standard analysis region, a value of zero be reported for BFR/BS. While this ignores the fact that MAR is reported as "missing data," meaning that BFR/BS can technically not be calculated, zero is a proper representation of the bone remodeling status, because the correct interpretation of the histomorphometric finding is that the absence of label, providing a zero MS/BS, overrides the technicality of not being able to calculate MAR. We recommend that when only single label is found in the standard analysis region, BFR/BS be reported as "missing data" for that patient. First, the patient's status will be properly represented in the MS/BS analysis. Second, because MAR cannot be calculated due to the lack of double label, that is actually what was observed. As for MAR, it is also permissible to calculate BFR/BS using an imputed value for MAR. When an imputed value for MAR is used, calculation of 
BFR/BS should be reported in both ways - one with BFR/BS as "missing data," and a second set using the imputed value of MAR to calculate BFR/BS. We recommend that when insufficient double labeled surface is present, the actual value for BFR/BS is reported, but flagged as based on insufficient double label evaluation and noted on the patient/animal report.

\section{Extended label search}

When no double label, or insufficient double label from which to make MAR measurements (see Section Extended label search) is found in the standard analysis regions, we recommend that a limited extended label search be undertaken to confirm the presence or absence of double label in the specimen, or identify additional double labels to solidify the measurement of MAR. The most important reason to conduct any form of extended label search is that when label is found outside the standard analysis regions, it can be confirmed that the subject complied with the labeling schedule. When an extended label search is undertaken, this should be noted on the patient's report.

The first place to examine is the trabecular bone outside the standard analysis regions defined as the area in sections outlined as in Fig. 1 to exclude the transitional zone and the bone fragments on the marrow edges in the two unstained sections that were analyzed (see Section Mineral apposition rate). If double label is found anywhere in this location, it can be assured that the patient took the fluorochrome label as directed and that, in a histomorphometric sense, some trabecular remodeling exists, despite the absence of double label in the standard region for histomorphometric analysis. This label can be used to solidify the measurement of MAR. The search is concluded.

If double label is not found as indicated earlier, the second place to examine is the cortices of the two unstained sections that contain the standard analysis regions. If double label is found in the cortices, it can be assured that the patient took both fluorochrome labels and has cortical remodeling, but not that the subject has trabecular remodeling. Cortical double labels cannot be used to solidify the measurement of MAR. It is just as certain that neither this limited search, nor any rational histomorphometric search, can ever confirm the absence of trabecular remodeling in the subject. In fact, experience has shown that most subjects, who lack double label in any part of the trabecular region from the first two levels of examination, have double label somewhere in the cortical region of those levels.

We believe that it is not warranted to continue to search for double labels in trabecular regions past this point. The discussion pertaining to the normal lower limit of remodeling provides a partial answer: total absence of double label anywhere in an iliac biopsy specimen did not occur in the standard analysis region of biopsies from a sizable cohort of normal premenopausal women in whom remodeling rates were quite low [8]. Thus, total absence of label in a specimen in an extended label search appears to mean that the subject's remodeling rate is abnormally low, provided one is reasonably confident that the subject complied with the labeling schedule. Again, it is the authors' contention that no search of the transilial biopsy specimen, including sectioning the specimen until exhaustion and examining repeated trabecular regions in detail, can assure the absence of trabecular remodeling. Our opinion is that it is certain that the finding of no double label in the standard analysis region indicates the existence of a low remodeling condition. The practical difference between this state and that indicated by sectioning a specimen until exhaustion and finding no double label, is too small to be worth the additional work. This finding of low remodeling is confirmed for a group when similar data emerge from multiple individuals within a treatment group. 
However, if either single or double label is found outside the standard analysis region during an extended label search, how does one express the result for purposes of analyzing data from a cohort? In this case it is justified to use MAR calculated from trabeculae outside the standard analysis region in calculation of those variables that use the value as long as this is noted. Further, as noted earlier, standard histomorphometric report forms should include a location that, in the event of a zero MS/BS, records that an extended label search was performed and its outcome.

Although compliance with the labeling instructions can sometimes be confirmed by collecting and analyzing urines for tetracycline at the time of labeling, there are practical problems with this, including sample collection, shipping and analytical difficulties. We recommend that a patient or research subject be contacted by telephone on the days when fluorochrome label is to be consumed, to review specific details (i.e., timing of tablets; not to be taken within $2 \mathrm{~h}$ of a meal; and not to be consumed with calcium supplements, dairy products, or antacids in the surrounding $2 \mathrm{~h}$ ) and obtain verification that the medication was properly taken. We also recommend that the investigators should: a) limit the time the specimen stays in aqueous fixative to $24 \mathrm{~h}, \mathrm{~b}$ ) limit the exposure of the specimen and cut sections to ambient light, and c) use appropriate UV light sources for fluorescence microscopy. For animals, we recommend that fluorochrome label be given by subcutaneous, rather than intraperitoneal (IP) injection. IP injections have the potential for injection into the gut or bladder which will result in lack of incorporation.

\section{Mineral apposition rate}

The mineral apposition rate (MAR, $\mu \mathrm{m} / \mathrm{day}$ ) is the rate at which mineral accretion occurs at a remodeling site during the period of bone formation. MAR is a fundamental histomorphometric variable that is the second one included in the calculation of all dynamic bone remodeling variables (i.e., bone formation rate, etc.) (Table 2). By itself, it is a reliable measure of osteoblast function. Further, its value is quite robust since its range of values is relatively small whenever its calculation is possible, provided there is no inhibition of mineralization.

There are at least three situations when its expression is problematic, i.e., when double label is absent in the standard analysis regions, but present on an extended label search; when insufficient double label is present in the standard analysis regions to provide a reliable assessment of MAR; and when double label is absent in both the standard analysis regions and extended label search. In the first case, one can report finding it in the extended label search, but regard it as missing data in the report, and derived variables should be reported as zero (i.e., BFR/BS and others, including MS/BS). When too few double labels were observed in the standard analysis region to provide reliable calculation of MAR, this should be noted in the report (see section Change in landscape). However, this MAR should be used in the calculation of derived variables. When no double label is found in either the standard analysis region or on extended label search, we recommend that MAR should be reported as missing data, and derived variables should be reported as zero.

Double label sites outside the standard analysis regions need not be added to the surface measurements and calculation of MS/BS and/or derived variables. That would create the impractical need to perform the entire set of static and dynamic histomorphometric measurements in all the outside areas examined in order to calculate derived variables. This would entail significant increases in time spent reading sections, and provide no useful contribution to the understanding of remodeling status. 


\section{Imputed value for MAR}

When single label is present in the standard analysis region and no double label or an unreliable amount of double label is found even on an extended label search, it is permissible to use an imputed value for MAR of $0.3 \mu \mathrm{m} / \mathrm{d}$, a figure reported as the lowest value seen in a cohort of human biopsies [9]. We recommend that when one reports data that uses an imputed value for MAR, one should report datasets both with and without the subjects in whom an imputed value was used. One should be aware that assigning an imputed value at the 5 th percentile of published normal MAR values $[9,10], 0.3 \mu \mathrm{m} / \mathrm{d}$, to any individual specimen in a group, creates a bias toward underestimating the true MAR and $\mathrm{BFR} / \mathrm{BS}$ for the group. On the other hand, if the absence of double label and MAR measurements is simply treated as missing data, the group central trend is biased toward overestimation.

\section{Adequate sample of double label width measurements for estimating MAR}

Inter-label width (iL.Wi) should be measured in any specimen that has trabecular double label anywhere in the standard analysis regions. A guideline for the minimum distance between measurement sites along a double label is $\sim 20 \mu \mathrm{m}$, but the selection of that distance is left to the individual investigator. Newer automatic histomorphometry software coupled with digital scanners that perform automatic measurements at intervals as small as $1 \mu \mathrm{m}$ are useful advances. The number of measurements taken (N.iL.Wi) and the number of double labels (N.dL) should be clearly recorded. In the reference cited earlier for healthy premenopausal women [8], the 10th, median and 90th percentiles for numbers of measurements of iL.Wi were 4.6,20, and 38, respectively. Thus, our recommendation is that when label is relatively scarce, a minimum of five measurements on a minimum of two independent double labeled surfaces be obtained. When ample label is present, sampling should be in the range of 20-40 measurements. Though all double labels should be thoroughly sampled, it is never necessary to obtain more than 50 individual measurements of iL.Wi to be used in the calculation of MAR. More than 50 measurements taken at $20 \mu \mathrm{m}$ intervals, does not result in significant change in the value (RR, unpublished data). When the minimum of five is not met for a subject, that fact should be noted on the subject/animal's report. Many histomorphometrists now use newer image analysis systems that perform the iL.Wi measurements automatically after the user outlines the borders of the area containing the double labels. The computer software then makes a large number of width measurements between the labels and expresses their average over all sites measured. The advantage with these systems is that each double label site is sampled many more times than can be done with older systems that require the reader to pick a small practical number of randomly chosen sites on which to perform individual measurements of iL.Wi. The number and length of the double label sites should be reported in order to estimate the adequacy of the sample of iL.Wi measurements.

\section{Adequate sampling of a human transilial biopsy (Fig. 1)}

The minimum acceptable total section area (Tt.Ar) in the standard analysis regions is 30 $\mathrm{mm}^{2}$. The minimum acceptable total section perimeter (Tt.Pm) is $60 \mathrm{~mm}$. The authors recommend obtaining two sets of sections as a standard. The first set is taken from an area a bit less than half way through the biopsy cylinder. The second is taken from an area $\sim 300$ $\mu \mathrm{m}$ further through the biopsy core. This leaves enough of the embedded block intact for additional sectioning if needed or for application of other analytical techniques (e.g., backscattered electron imaging to assess mineralization density).

Each set consists of three sections, one for Goldner's stain (Fig. 1), one for Toluidine blue (or McNeal's tetrachrome) stain and one left unstained for fluorescent microscopy. If the 
two levels do not provide standard analysis regions with at least $30 \mathrm{~mm}^{2}$ of total section area (Tt.Ar) and $60 \mathrm{~mm}$ of total section perimeter (Tt.Pm), another set is taken $\sim 300 \mu \mathrm{m}$ further through the specimen, and more measurements are added to the dataset for that specimen. It is rare that an intact, well-fixed human iliac biopsy core taken with a $7.5 \mathrm{~mm}$ trephine contains less than $30 \mathrm{~mm}^{2}$ in the standard analysis regions from two sets of sections.

\section{Activation frequency and erosion depth: meaning and measurement}

Bone remodeling or turnover is carried out by teams of juxtaposed osteoclasts (at the front) and osteoblasts (bringing up the rear), comprising temporary anatomical structures known as basic multicellular units (BMUs). In cortical bone, the BMUs drill tunnels or "cutting cones" through the compact tissue while in spongy, cancellous bone, they usually gouge across the trabecular surface forming a serpentine trench [11]. Bone turnover begins by conversion of a quiescent skeletal surface to a remodeling site, a process referred to as activation. The activation frequency (Ac.f) represents the probability (\#/year) that a new remodeling cycle will be initiated at any point on the cancellous perimeter and is calculated by dividing the surface-based bone formation rate (BFR/BS) by the average amount of bone formed by a team of osteoblasts per activation event, i.e., the wall thickness (W.Th). Thus, Ac.f $=((\mathrm{BFR} /$ $\mathrm{BS} / \mathrm{W}$.Th). The measurement is more than just the rate of BMU origination, as it corresponds to the product of the frequency of BMU origination and completed bone structural unit (BSU) thickness, rate of progression and lifespan. Ac.f is the best available two-dimensional histologic index of the intensity of bone remodeling or turnover and as such, also represents the number of BMUs currently active.

Ac.f is a highly derived variable whose determination presents issues in calculation (Table 2), assumptions, and interpretation. As discussed earlier, fluorescent labels are occasionally missing. In addition, even without an increase in bone formation rate, Ac.f will increase if W.Th decreases, as it does with age [12], and as it perhaps does with some anti-remodeling therapies. If W.Th decreases without a change in BFR/BS, Ac.f could increase even though the "rate of bone turnover" assessed by BFR/BS is apparently unchanged. Furthermore, interpretation of the Ac.f becomes complicated when an anabolic therapy increases wall thickness or thickens a trabecular profile by adding bone to a previously completed BSU, as occurs with the intermittent administration of parathyroid hormone. Because the calculation of Ac.f is based on measurements of MS/BS, it does not measure directly the activation of osteoclasts, the vigor of their work, or their lifespan.

Some prefer using just MS/BS alone as a measure of the bone remodeling rate. However, this approach has drawbacks: it is influenced greatly by the surface density and volume of existing trabeculae, and does not take into account the length of time required to complete the formation phase of remodeling sites. It is, however, a valid expression of remodeling rates, and is correlated with remodeling as expressed by measurement of circulating biochemical markers of bone formation and resorption [13]. As mentioned earlier, MS/BS is also robust even in the face of absence of double and single label, as zero is a reasonable value for MS/BS in such specimens.

While bone histomorphometric techniques work very well for the evaluation of bone formation status, they are less reliable when used to evaluate bone resorption. Though the concept of erosion depth is important and valid, there are multiple practical problems associated with its measurement. Erosion depth represents an index of osteoclast vigor that is otherwise difficult to determine. In theory, erosion cavities have an adverse effect on cancellous bone strength disproportionate to the decrease in bone mass that the cavities represent [14]. Furthermore, loss of bone strength due to perforation of trabecular profiles becomes more likely as erosion depth increases. Erosion depth has a wide frequency of 
distribution. Limited evidence indicates that it decreases with age more noticeably in women than in men [15]. Difficulty with the measurement itself occurs when erosion cavities have unusual shapes that defy efforts to reconstruct the original bone perimeter [16]. In addition, erosion cavities are often few and inconspicuous in specimens obtained from elderly patients with osteoporosis. Selection of only the cavities ideal for measurement may cause considerable measurement bias [17].

Eroded surface is a static measure that gives a valid snapshot of the morphologic picture of a specimen's resorption surfaces, but no direct information concerning the dynamic status of resorption $[13,18]$, because rate of osteoclast activity cannot be directly measured. The eroded surface is composed of the osteoclast surface (about $1 \%$ of the trabecular bone surface in humans) plus the reversal surface (about $9 \%$ of the trabecular bone surface in humans). Osteoclast surface has proven useful in identifying the occasional occurrence of large osteoclasts in persons treated with bisphosphonates $[19,20]$. Reversal surfaces increase with defective or delayed bone formation, as occurs with glucocorticoid excess, and thus may have little to do with the current amount of bone resorption. Moreover, most antiresorptive drugs decrease the ability of osteoclasts to erode bone, reduce the BFR, and actually increase the reversal surface, thereby, increasing eroded surface. Thus the interpretation of eroded surface in cases in which anti-remodeling drugs have been used can be misleading.

We recommend that osteoclast surface be included, because there is good inter-laboratory consensus on its identification and it can provide valuable information on cell number. We also recommend that despite limitations, eroded surface be included. While we acknowledge the concept of erosion depth, we recommend that it not be evaluated in transilial biopsies (Table 3), because the sample is usually small, the measurement can be quite variable, and no inter-laboratory consensus on the optimal method for obtaining erosion depth data exists.

\section{Histomorphometric variables (Tables 1-3)}

The variables, and their symbols, included in Tables 1-3 are taken from the 1987 reference that standardized the nomenclature, symbols and units for bone histomorphometry [3].

We recommend dividing histomorphometric variables into two classifications: measured variables (Table 1) and variables that should be reported (Table 2) in all publication of histomorphometric studies. However, the four cortical measurements can be omitted in any report that does not require assessment of cortical remodeling. The reported variables include a few measured variables used for quality assurance and a number of others that are calculated by previously published formulae that are recorded here. Since microscope variables refer to data that are collected from two-dimensional sections, they are always expressed in two dimensions. Since reported variables nearly always refer to calculations that are done to understand bone remodeling in a three-dimensional sense, they are expressed in three dimensions (3D). We feel that the variables listed in Table 2 are necessary and sufficient to show the complete picture of the histomorphometric findings in any human or animal experiment.

The thickness expressions in the calculated trabecular variables are converted to 3D by multiplying the width (microscope) measurements by $\pi / 4$, the obliquity correction factor [3]. It is not necessary for cortical bone. Converting to 3D may aid in understanding the dynamic (tetracycline-based) histomorphometric expressions of remodeling, and correlation with non-histomorphometric measurements of dynamic bone remodeling and/or 3D measurements of micro-architecture using $\mu \mathrm{CT}$ may be improved [21]. We recommend that authors indicate whether the obliquity correction factor was used in order to compare with data from other reports. 
When widths $(\mathrm{O} . \mathrm{Wi}, \mathrm{W} . \mathrm{Wi}$, etc.) are measured at the microscope, it is important to report the number of width measurements of each type to obtain an average value (i.e., N.O.Wi). We consider the collection of some of the data in Table 1 optional and have so labeled them.

All variables in Table 2 should be included in reports and papers. As mentioned earlier, when imputed MAR is applied for any member of a group, the group data should be reported both with the imputed MAR data, and with MAR treated as missing data. The cortical thickness measurements have gained in importance recently as investigators attempt to understand the contribution of cortical bone to risk of fracture and the effect of therapeutic agents that reduce remodeling and cortical porosity.

The variables in Table 3 can be calculated and may be reported. It is our experience that these variables are problematic. In some cases, their measurement is difficult and has a tendency to be biased. For cellular endpoints, inconsistent fixation may be a problem. In other cases (Tb.Th, Tb.N, Tb.Sp), better methods for their measurement exist, such as MicroCT, in which their values are calculated from 3D reconstructions. Fibrosis surface is a good index, but is only relevant for a few conditions. The periods (F.P, R.P, and Rm.P) and BFR/TV are heavily derived variables that have been of limited use in understanding how drugs work to reduce fracture rate in osteoporosis.

There are numerous additional histomorphometric variables that could be named [3] and added to Tables 1 and 2. However, the main use for histomorphometry is to understand the status of bone remodeling and mineralization. The variables in Tables 1 and 2 are those that are fundamental to that understanding, and thus justifying their inclusion.

Examples of measured variables used in other types of histomorphometric examinations include: Number of osteocyte lacunae per unit B.Ar, number/fraction of osteocyte lacunae containing normal osteocytes, number/fraction of osteocyte lacunae containing no osteocytes; number/fraction of osteocyte lacunae containing apoptotic osteocytes, number/ fraction of osteocytes that are apoptotic, number of apoptotic osteoblasts/B.Pm, and area of woven bone/B.Ar. The publication on nomenclature [3] suggests the possibility of numerous useful calculated variables derived from the above microscope variables and others. We recommend that when needed to suit a particular examination, histomorphometrists report additional variables that are clearly-explained and appropriate for the purposes of the specific investigation.

\section{Application of bone histomorphometry to animal models}

When properly used, animal models for bone research, most notably for pharmacology studies, are considered among the most predictive models for human outcomes in any field of pre-clinical pharmacology [22]. Adult rodents (mice and rats) and adult large animals (e.g., non-human primates [NHPs: cynomolgus and rhesus], canines, sheep, etc.) are differentiated by the fact that only cortical bone of adult large animals displays Haversian remodeling like adult human cortical bone. Cortical bone of adult rodents has no ambient Haversian bone remodeling. [23,24]. Thus, the adult rodent is best used only as a model of trabecular bone remodeling.

Pre-necropsy dual fluorochrome labeling (subcutaneous calcein injection) with a five-toseven day interlabel time period for adult rats and a two-to-five day interlabel time period for mice are routinely applied to produce double labels similar to those seen in humans. The adult ovariectomized (OVX) rat [25] is commonly used in pre-clinical pharmacology studies. Mice exhibit strain-related variation in bone behavior. They are widely-used today to study variation in bone phenotype due to genetic alterations. Adult NHPs aged 10-12 years display estrogen-deficiency-induced bone loss and are used both to evaluate cortical 
bone remodeling and study bone responses in the typical human osteoporotic fracture sites. Adult canines have appropriate trabecular and cortical bone remodeling, but do not display reliable estrogen deficiency-related bone loss.

Most variables used in the analysis of trabecular bone in transilial bone biopsy specimens from humans (Tables 1 and 2) not only can be measured/analyzed, but also have similar meaning in adult rodents. The exception is W.Wi. Though it can be evaluated in adult rodent vertebral trabecular bone, W.Wi cannot be readily measured in long bone metaphyseal trabecular bone because of the paucity of completed bone structural units. Fortunately, an excellent understanding of the effects of various agents (estrogens, bisphosphonates, PTH, etc.) and physiologic conditions (e.g., skeletal unloading, vitamin D deficiency, etc.) can be obtained in rodent experiments without evaluating W.Wi, by relying on MS/BS, BFR/BS, and related variables. Eroded perimeter (E.Pm) is considered more difficult to identify in rodents than in humans, making it less reliable than in humans. Therefore, osteoclast surface (Oc.S/BS) is recommended as the best index of bone resorption in rodents. Reversal perimeter, fibrosis perimeter, and erosion depth are generally not evaluated in rodents. Mineralization lag time is much shorter in rodents than in humans, resulting in very thin osteoid seams. Therefore, some variables such as osteoid thickness and volume are rarely reported in rodent studies, unless an agent impairs bone mineralization and induces osteoid accumulation. We recommend that MS/BS, MAR, and BFR/BS be handled as in humans with one exception. When double fluorochrome label is absent, since the imputed value for MAR $(0.3 \mu \mathrm{m} /$ day $)$ discussed earlier was derived from human data; its use in animal studies is not recommended. We recommend that imputed/assigned MAR values for rodents not be used until an imputed MAR value derived specifically from rodents has been established.

The anatomical sites most frequently used for histomorphometric study in adult rats are the proximal tibial and distal femoral metaphyses. When the metaphyses are too osteopenic, something that is more likely to occur after ovariectomy and with advancing age, the lumbar vertebral body can and should be substituted. The region of interest for histomorphometric measurements intended to provide data that address adult human bone remodeling should always exclude the primary spongiosa adjacent to the growth plate, which is composed of newly-formed juvenile bone that compares poorly to adult human bone. The anatomical sites most frequently used in adult mice to provide data about adult human bone remodeling are the distal femoral metaphysis, proximal tibial metaphysis, and L1-L4 vertebral bodies. As for rats, when the metaphyses are too osteopenic (e.g., after ovariectomy and in aged mice), the lumbar vertebral body can be substituted. These sites are smaller, but generally more uniform, than regions of the human transilial biopsy specimen. The minimum acceptable Tt.Ar in rat trabecular bone is approximately $6-8 \mathrm{~mm}^{2}$, while the minimum acceptable B.Pm is approximately $25 \mathrm{~mm}$, both less than in the human. Histomorphometric measurements are usually performed in two sections froma skeletal site separated by at least $50 \mu \mathrm{m}$. The minimum acceptable Tt.Ar and B.Pm in mouse trabecular bone is approximately $60 \%$ of that in rats, due to the smaller size of murine bones. Cortical bone studies in rodents are done in cross-sections taken at either the mid-point of the femur or the region located 1-2 mm proximal to the tibiofibular junction. These areas are used for the evaluation of periosteal and endocortical surface endpoints. The internal cortex of rodents cannot be used for the evaluation of Haversian remodeling. When conducting rodent experiments involving bone, it is better to use large group sizes, rather than analyze more section area and perimeter froma small number of animals. Although we recommend minimum group sizes of 6-8 rodents for bone histomorphometric studies as a starting point, it is best for group sizes in individual experiments to be determined by a power analysis.

The anatomical sites most frequently evaluated in adult non-human primates (NHP) are the typical human osteoporotic fracture sites, the vertebral body and the proximal femur. Since 
these sites can never be sampled systematically in experiments involving living humans, most investigators feel that studying these sites leads to the most direct understanding of how agents work at osteoporotic fracture sites in humans. The NHP iliumis also used for a direct comparison to the usual sampling site in humans. The osteoporotic fracture sites in NHPs yield enough trabecular bone that the minimums for sampling the human transilial biopsy specimen apply. On the other hand, the NHP ilium generally yields a smaller trabecular bone sample than the human transilial biopsy specimen. Its minimum acceptable Tt.Ar is approximately $8 \mathrm{~mm}^{2}$, while its minimum acceptable B.Pm is approximately 25 $\mathrm{mm}$. Multiple sections at $250 \mu \mathrm{m}$ intervals should be studied to assure that these minimums are met. Cortical bone studies of periosteal and endocortical surface endpoints in NHPs are done using cross-sections taken at the seventh rib, the mid-point of the femur, the mid-point of the femoral neck, and the center of the lumbar vertebral body. The interior of the cortex at these sites is used to evaluate Haversian remodeling. When doing NHP experiments that involve bone, the group size is often enlarged to accommodate mechanical testing of the osteoporotic fracture sites, providing a generous sample size for histomorphometry.

\section{Extrapolating data from a transilial biopsy specimen to the whole skeleton}

The trabecular bone of the ilium is a red marrow site with a remodeling rate of $\sim 10-15 \%$ / year. When considering bone remodeling activity, it appears to be representative of similar red marrow skeletal sites, such as the vertebral body [26]. It is not representative of fatty marrow sites with their low remodeling rates. Its relationship to the proximal femur has not been studied in any detail.

Transilial bone biopsies are not the best way to evaluate bone mass. While BV/TV in large groups of individuals may actually give reasonable data and be useful in the experimental setting, the small sample for analysis is a problem. DXA (dual energy X-ray absorptiometry) and CT (computed tomography) not only sample much larger areas, but also work in the osteoporotic fracture sites and are non-invasive.

We do not believe the Haversian portion of the cortical bone of a transilial biopsy specimen can be sampled in a manner that allows calculation of reliable bone histomorphometric data, because of the problems with consistent orientation during sectioning of the specimen. The periosteal and endocortical surfaces of the two cortices are suitable for study, but tend to provide limited perimeter. The data from each of the two cortices should be collected and reported separately.

\section{Defining "anabolic" in histomorphometric terms}

Conceptually, bone anabolism requires a significant increase in bone mass that is driven by an increased rate of formation of new bone unaccompanied by an offsetting increase in the rate of bone resorption. This increase in bone mass can occur by formation activity at trabecular, endocortical, or periosteal surfaces. For osteoporotic patients, the bone mass increases should occur at the typical osteoporotic fracture sites, such as the spine, the hip, and the wrist. It is noteworthy that this concept, and/or definition, of "bone anabolism" does not apply in a growing animal model since bone anabolism occurs without any treatment during growth.

In a technical sense, a bone anabolic agent is one that, in comparison to a baseline state or a placebo group, increases bone mass by elevating the bone formation rate mainly through increasing mineralizing surface and osteoblast number, rather than through increasing activity of osteoblasts. Bone histomorphometric data are necessary, but not sufficient to identify such agents properly. Likewise, non-invasive measurements of bone mass are necessary, but not sufficient. The two types of measurements must be considered together. 
In humans, bone histomorphometric examination of transilial biopsies is the method of choice for proving that bone formation has been stimulated. In small animals, sites such as the proximal tibial metaphysis or vertebral body cancellous bone are studied. For both animals and humans, increases in tissue level endpoints such as W.Th, MS/BS, OS/BS, and BFR/BS form the cornerstone for recognizing higher levels of bone formation. They signal the participation of increased numbers of osteoblasts and the existence of more sites of bone formation. MAR only defines the status of osteoblast activity. Documenting higher MAR alone is not sufficient to define anabolism. Mineralization status should remain normal in the presence of an anabolic agent. Mineralization status is best defined by appropriate values for variables data such as Mlt, Omt, and MS/OS. Increases in OS/BS must be accompanied by appropriate increases in MS/BS. Biochemical markers of bone formation are often used to confirm bone histomorphometric findings regarding bone formation status, but lack the reliability to be used alone for this purpose.

The best way to measure anabolic change in bone mass is a non-invasive method that samples large amounts of mineral, preferably at osteoporotic fracture sites. Bone histomorphometry produces variables such as BV/TV and Ct.Ar that are indices of the bone mass of the specimen itself. However, the specimen represents but a small sample of the skeleton that may not reflect the status of osteoporotic fracture sites in humans or the spine of experimental animals for bonemass purposes.

The best known methods for evaluating bone mass are DXA and CT. One or both should always be applied in both human and animal experiments to determine bonemass, because of their superior sampling ability, and, in humans, their ability to evaluate the osteoporotic fracture sites and do repeat measurements.

\section{Warning: pseudoanabolic agents}

Frequently, agents display only one feature of the two required to define an anabolic agent. Agents are known that cause rises in bone mass to occur in the absence of increased bone formation rate. Others are known that induce bone formation rises without an accompanying increase in bone mass. Neither type of agent is anabolic.

Anti-remodeling agents cause bone mass increases soon after they are begun. The rate of initiation of new bone remodeling units declines to a new and lower level, while previouslyactivated bone remodeling units finish their formation phases. Bone mass rises, due to decreased bone resorption in the presence of bone formation rate sustained at its previous rate, as the "remodeling space" is filled in. Bone formation rate eventually declines during long-term administration of anti-remodeling agents, because the activation frequency of new remodeling units has permanently declined. Examples include estrogens, bisphosphonates, and anti-RANK Ligand antibodies. These are not anabolic agents.

Agents that stimulate remodeling raise bone formation rates soon after they are begun. Early after the rate of initiation of new bone remodeling units rises, more are in the resorption phase than are finishing the formation phase, actually creating bone loss. Bone mass declines, due to a greater rate of bone remodeling that increases the amount of unfilled "remodeling space." Bone formation rate stays high and bone mass settles at a lower level during long-term administration of remodeling stimulating agents because the remodeling space is permanently enlarged. Examples include thyroxine and growth hormone. These are not anabolic agents. 


\section{Defining and detecting "oversuppression" of remodeling}

The bone remodeling rate ordinarily remains within in a certain range that maintains health of the skeleton. It is measurable by bone histomorphometric techniques and, to some degree, by biochemical markers for formation and resorption. High remodeling rates, as in postmenopausal women, patients with primary hyperthyroidism, and some cases of renal osteodystrophy, cause bone loss, decreased bone strength, and increased fracture risk. These elevated rates have been measured by both biochemical markers of formation and resorption and bone histomorphometric techniques. The well-known age-related rise in fracture risk is associated with increased remodeling as expressed by activation frequency, particularly around menopause. It is, thus, not surprising that anti-remodeling agents work by a tissuelevel mechanism that decreases activation frequency, and reduces fracture risk.

Low bone remodeling rates represent the other side of that coin. It must also be remembered that defining and detecting "oversuppression" of remodeling are two very different tasks. In theory, oversuppression is defined as a condition (perhaps caused by a pharmacologic intervention) that results in levels of remodeling activity low enough to cause harm to the individual in the form of increased risk of fracture, much in the same way that high levels of remodeling activity are associated with increased risk of fracture. Though speculation about the existence of ultra-low remodeling rates that might be called oversuppression has emerged recently, no skeletal adverse consequences have been linked convincingly to such ultra low remodeling rates. In the absence of a clear relationship of low bone remodeling rate to adverse outcomes, we feel that it is difficult to define a specific value for rate of bone remodeling that can be considered "oversuppression." That being said, we further believe that the detection of a low remodeling state is problematic, given the tools available for judging remodeling rates. As we have stated, while transilial bone biopsy-based histomorphometry can be used to detect a range of bone remodeling activity, when BFR/BS and Ac.f are reported as zero, the correct interpretation is that remodeling is low, but not necessarily, or not even likely, zero, anywhere in either the specimen or the skeleton. Furthermore, individuals who have BFR/BS and Ac.f reported as zero tend to display a range of bone biochemical marker values from normal to low. Importantly, the biochemical marker values rarely or never reach zero.

One method of analysis that can be used when there are no fluorochrome labels in a biopsy specimen is illustrated in Table 4. We have recommended that a finding of no double label in the standard sampling areas of an individual patient always be taken as a certain signal of a low remodeling state, but never be taken to mean that the patient completely lacks remodeling. We have also recommended that the main method for presenting the data from such patients in studies with multiple treatment groups, is that they be included for MS/BS (value of zero) and BFR/BS (value of zero) when no label of any kind is found, but excluded from other important calculations such as mineral apposition rate (MAR) and all others that depend upon MAR, due to the inability to calculate MAR. Excluding non-double label patients from calculations of central tendency, creates an inaccurate upward bias in the group BFR/BS and Ac.f values (less likely for MAR because it is so constant), in groups containing patients without double label.

There is a way to compensate partially for this bias. The number of patients in a group excluded from MAR and BFR/BS calculation due to lack of double label can serve as a useful index of group remodeling state. Based on published data [8,27], investigators can and should determine whether a treatment group has more subjects without double label than: a) any logical comparator group within the study, b) healthy premenopausal women [8], and c) untreated post-menopausal osteoporotic women [27]. 
In Table 4, we present group sizes and the minimum numbers of subjects who lack double label in the test group to establish significant differences $(\mathrm{P}<.05$ and $\mathrm{P}<.01)$. We also add a comparison that uses typical group sizes for pre-clinical studies. When the difference is significant, a significantly low remodeling state exists in the test group. We use a typical group size of 30-32 for human osteoporosis studies. For example, it is of interest to know whether a treated group has lower bone formation rate than healthy pre-menopausal women. If three subjects have no double label, remodeling rate is likely to be significantly lower than in premenopausal women $(\mathrm{P}<.05)$. If five subjects lack double label, remodeling rate is even more likely to be significantly lower than in premenopausal women $(\mathrm{P}<.01)$. We use the group sizes presented $[8,27]$ for the published data. This analysis was done with the Chi Square test, and can be performed similarly for any comparator group size.

\section{Acknowledgments}

Recker: Consultant for Merck, Lilly, Wyeth, Procter \& Gamble, Amgen, Roche, Glaxo Smith Kline, Novartis, and NPS Allelix; and grant/research support from Merck, Lilly, Wyeth, Procter\&Gamble, Amgen, Roche, Glaxo Smith Kline, Novartis, NPS Allelix, and Sanofi-Aventis through institutional grants.

Weinstein: Supported by a VA Merit Review Grant from the Office of Research and Development Department of Veterans Affairs and the National Institutes of Health (P01-AG13918).

Wronski: Research support from Amgen and Lexicon Pharmaceuticals.

Kimmel: None.

Dempster: None.

Burr: Grant/Research Support, Consultant, Scientific Advisor, Speaker: Eli Lilly, Procter and Gamble, Alliance for Better Bone Health, Pfizer, NephroGenex, Amgen, PharmaLegacy.

The authors wish to thank Roger Phipps for several discussions about the calculation and interpretation of the mineral apposition rate that helped clarify our thinking.

\section{References}

1. Frost HM, Villanueva AR, Roth H. Measurement of bone formation in a 57 year old man by means of tetracyclines. Henry Ford Hosp Med Bull. 1960; 8:239-54. [PubMed: 13825401]

2. Recker, RR., editor. Bone histomorphometry: techniques and interpretation. Boca Raton: CRC Press; 1983.

3. Parfitt AM, Drezner MK, Glorieux FH, Kanis JA, Malluche H, Meunier PJ, et al. Bone histomorphometry: standardization of nomenclature, symbols, and units. J Bone Miner Res. 1987; 2:595-610. [PubMed: 3455637]

4. Recker RR, Delmas PD, Halse J, Reid IR, Boonen S, Garcia-Hernandez PA, et al. Effects of intravenous zoledronic acid once yearly on bone remodeling and bone structure. J Bone Miner Res. 2008; 23:6-16. [PubMed: 17892374]

5. Recker RR, Weinstein R, Chesnut CH III, Schimmer RC, Mahoney P, Hughes C, et al. Histomorphometric evaluation of daily and intermittent oral ibandronate in women with postmenopausal osteoporosis: results from the BONE study. Osteoporos Int. 2004; 15:231-7. [PubMed: 14727011]

6. Schwartz MP, Recker RR. The label escape error: determination of the active bone-forming surface in histologic sections of bone measured by tetracycline double labels. Metab Bone Dis Relat Res. 1982; 4:237-41. [PubMed: 7182723]

7. Keshawarz NM, Recker RR. The label escape error: comparison of measured and theoretical fraction of total bone-trabecular surface covered by single label in normals and patients with osteoporosis. Bone. 1986; 7:83-7. [PubMed: 3087397] 
8. Recker R, Lappe J, Davies KM, Heaney R. Bone remodeling increases substantially in the years after menopause and remains increased in older osteoporosis patients. J Bone Miner Res. 2004; 19:1628-33. [PubMed: 15355557]

9. Foldes J, Shih MS, Parfitt AM. Frequency distributions of tetracycline-based measurements: implications for the interpretation of bone formation indices in the absense of double-labeled surfaces. J Bone Miner Res. 1990; 5:1063-7. [PubMed: 2080717]

10. Hauge E, Mosekilde L, Melsen F. Missing observations in bone histomorphometry on osteoporosis: implications and suggestions for an approach. Bone. 1999; 25:389-95. [PubMed: 10511104]

11. Weinstein RS, Manolagas SC. Apoptosis and osteoporosis. Am J Med. 2000; 108:153-64. [PubMed: 11126309]

12. Recker RR, Kimmel DB, Parfitt AM, Davies KM, Keshawarz N, Hinders S. Static and tetracycline-based bone histomorphometric data from 34 normal postmenopausal females. J Bone Miner Res. 1988; 3:133-44. [PubMed: 3213608]

13. Hernandez CJ. How can bone turnover modify bone strength independent of bone mass? Bone. 2008; 42:1014-20. [PubMed: 18373970]

14. van der Linden JC, Homminga J, Verhaar JAN, Weinans H. Mechanical consequences of bone loss in cancellous bone. J Bone Miner Res. 2001; 16:457-65. [PubMed: 11277263]

15. Eriksen EF, Mosekilde L, Melsen F. Trabecular bone resorption depth decreases with age: differences between normal males and females. Bone. 1985; 6:141-6. [PubMed: 4027092]

16. Fihn SD, Johnson C, Roberts PL, Running K, Stamm WE. Trimethoprim-sulfamethoxazole for acute dysuria in women: a single-dose or 10-day course. Ann Intern Med. 1988; 108:350-7. [PubMed: 3257670]

17. Cohen-Solal M, Shih M-S, Lundy M, Parfitt A. A new method for measuring cancellous bone erosion depth: application to the cellular mechanisms of bone loss in postmenopausal osteoporosis. J Bone Miner Res. 1991; 6:1331-8. [PubMed: 1792944]

18. Allen MR, Erickson AM, Wang X, Burr DB, Martin RB, Hazelwood SJ. Morphological assessment of basic multicellular unit resorption parameters in dogs shows additional mechanisms of bisphosphonate effects on bone. Calcif Tissue Int. 2010; 86:67-71. [PubMed: 19953232]

19. Weinstein RS, Roberson PK, Manolagas SC. Giant osteoclast formation and long-term oral bisphosphonate therapy. N Engl J Med. 2009; 360:53-62. [PubMed: 19118304]

20. Recker RR. Should we be concerned about giant osteoclasts? BoneKEy. 2009; 6:157-8.

21. Akhter MP, Lappe JM, Davies KM, Recker RR. Transmenopausal changes in the trabecular bone structure. Bone. 2007; 41:111-6. [PubMed: 17499038]

22. Kimmel, DB. Animal models in osteoporosis Research. In: Rodan, G.; Bilizekian, J.; Raisz, L., editors. Principles of Bone Biology. San Diego: Academic Press; 2001. p. 543-64.

23. Erben RG. Trabecular and endocorticl bone surfaces in the rat: modeling or remodeling? Anat Rec. 1996; 246:39-46. [PubMed: 8876822]

24. Frost HM, Jee WSS. On the rat model of human osteopenias and osteoporoses. Bone Miner. 1992; 18:227-36. [PubMed: 1392696]

25. Wronski TJ, Cintron M, Dann LM. Temporal relationship between bone loss and increased bone turnover in ovariectomized rats. Calcif Tissue Int. 1988; 43:179-83. [PubMed: 3141020]

26. Mellish RWE, Ferguson-Pell MW, Cochran GVB, Lindsay R, Dempster DW. A new manual method for assessing two-dimensional cancellous bone structure: comparison between iliac crest and lumbar vertebra. J Bone Miner Res. 1991; 6:689-96. [PubMed: 1950674]

27. Kimmel DB, Recker RR, Gallagher JC, Vaswani AS, Aloia JF. A comparison of iliac bone histomorphometric data in post- menopausal osteoporotic and normal subjects. Bone Miner. 1990; 11:217-35. [PubMed: 2268749] 


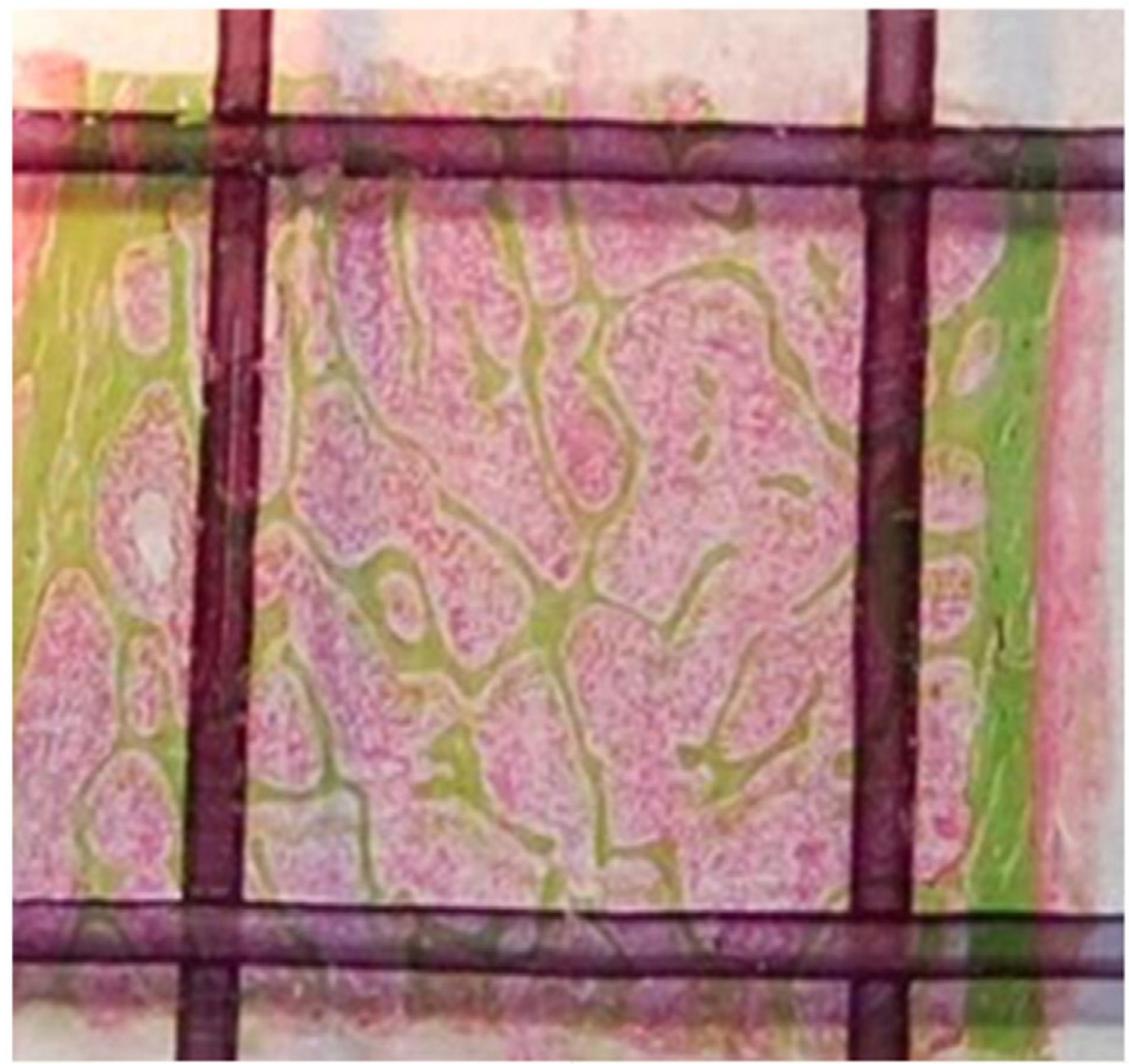

Fig. 1.

Photomicrograph is displayed from a section $6 \mu \mathrm{m}$ in thickness from one level of a transilial biopsy specimen. The internal and external cortices and the intervening trabecular region ( 10x magnification) are apparent. Modified Goldner staining has been completed. Black lines delineate a typical standard analysis region with dimensions $\sim 4 \mathrm{~mm}$ (horizontal) by 5.5 $\mathrm{mm}$ (vertical). Note that the transitional zone adjacent to each cortex is excluded by the vertical lines. Similarly, the periphery of the core that may include tissue damaged by the wall of the trephine is excluded by the horizontal lines. All trabecular tissue inside the lines is evaluated. Most microscope data (Table 1) are collected from a Goldner or similarly stained slide. A very similar analysis region from the unstained section is outlined and measured only for dL.Pm, sL.Pm, dL.N, sL.N, B.Pm, iL.Wi, and N.iL.Wi. 
Table 1

Measured variables.

\begin{tabular}{|c|c|c|c|}
\hline Variable & Units & Symbol & Definition \\
\hline \multicolumn{4}{|l|}{ Trabecular region } \\
\hline Tissue area measured & $\mathrm{mm}^{2}$ & Tt.Ar & Total trabecular tissue area of sections examined \\
\hline Bone area & $\mathrm{mm}^{2}$ & B.Ar & Total area of trabecular bone silhouettes examined (includes osteoid) \\
\hline Osteoid area & $\mathrm{mm}^{2}$ & O.Ar & Total area of osteoid examined \\
\hline Osteoid width & $\mu \mathrm{m}$ & O.Wi & Average width of osteoid seams \\
\hline Number of osteoid widths measured & \# & N.O.Wi & Number of osteoid width measurements \\
\hline Bone perimeter & $\mathrm{mm}$ & B.Pm & Total length of bone perimeter examined \\
\hline Wall width & $\mu \mathrm{m}$ & W.Wi & Average of osteon wall width measurements \\
\hline Number of wall widths measured & $\#$ & N.W.Wi & Number of wall width measurements \\
\hline Double label perimeter & $\mathrm{mm}$ & dL.Pm & Total length of double label perimeter examined \\
\hline Single label perimeter & $\mathrm{mm}$ & sL.Pm & Total length of single label perimeter examined \\
\hline Osteoid perimeter & $\mathrm{mm}$ & O.Pm & Total length of osteoid perimeter examined \\
\hline Eroded perimeter & $\mathrm{mm}$ & E.Pm & Total length of eroded (resorption) perimeter examined \\
\hline Osteoclast perimeter & $\mathrm{mm}$ & Oc.Pm & Total length of perimeter occupied by osteoclasts \\
\hline Osteoblast perimeter & $\mathrm{mm}$ & Ob.Pm & Total length of perimeter occupied by osteoblasts \\
\hline Reversal perimeter $^{a}$ & $\mathrm{~mm}$ & Rv.Pm & Total length of perimeter occupied by reversal surface \\
\hline Fibrosis perimeter & $\mathrm{mm}$ & $\mathrm{Fb} . \mathrm{Pm}$ & Total length of perimeter occupied by fibrous surface \\
\hline Osteoclast number & $\mathrm{mm}$ & Oc.N & Total number of osteoclasts \\
\hline Interlabel width & $\mu \mathrm{m}$ & iL.Wi & Average width between labels \\
\hline Number of interlabel widths measured & $\#$ & N.iL.Wi & Number of interlabel width measurements \\
\hline Double label number & $\#$ & dL.N & Total number of discrete double labels examined \\
\hline Single label number & $\#$ & sL.N & Total number of discrete single labels examined \\
\hline Erosion depth ${ }^{a}$ & $\mu \mathrm{m}$ & E.D & Average depth of active resorption lacunae \\
\hline Number of erosion depths measured ${ }^{a}$ & \# & N.E.D & Number of erosion depth measurements \\
\hline \multicolumn{4}{|l|}{ Cortical regions ${ }^{b}$} \\
\hline Cortex \#1 width & $\mu \mathrm{m}$ & Ct1.Wi & Total width of Cortex\#1 \\
\hline Number of cortex \#1 widths measured & $\#$ & N.Ct1.Wi & Number of Cortex\#1 width measurements \\
\hline Cortex \#1 area & $\mathrm{mm}^{2}$ & Ct1.Ar & Total area occupied by Cortex\#1 \\
\hline Cortex \#1 pore area & $\mathrm{mm}^{2}$ & Ct1.Po.Ar & Total area occupied by Cortex\#1 pores \\
\hline Cortex \#2 width & $\mu \mathrm{m}$ & $\mathrm{Ct} 2 . \mathrm{Wi}$ & Total width of Cortex\#2 \\
\hline Number of Cortex \#2 widths measured & $\#$ & N.Ct2.Wi & Number of Cortex\#2 width measurements \\
\hline Cortex \#2 area & $\mathrm{mm}^{2}$ & $\mathrm{Ct} 2 . \mathrm{Ar}$ & Total Area occupied by Cortex\#2 \\
\hline Cortex \#2 Pore area & $\mathrm{mm}^{2}$ & $\mathrm{Ct} 2 . \mathrm{Po} . \mathrm{Ar}$ & Total area occupied by Cortex\#2 pores \\
\hline
\end{tabular}

Bone. Author manuscript; available in PMC 2012 November 1. 
Table 2

Recommended variables to report.

\begin{tabular}{|c|c|c|c|}
\hline Variables & Units & Symbol & Definition/formula \\
\hline Tissue area & $\mathrm{mm}^{2}$ & Tt.Ar & Area of sections examined \\
\hline Bone area & $\mathrm{mm}^{2}$ & B.Ar & Area of trabecular silhouettes examined \\
\hline Bone perimeter & $\mathrm{mm}$ & B.Pm & Total length of perimeter examined \\
\hline Bone volume & $\%$ & $\mathrm{BV} / \mathrm{TV}$ & Percent of marrow space occupied by bone $=100 *(\mathrm{~B} . \mathrm{Ar} / \mathrm{Tt} \cdot \mathrm{Ar})$ \\
\hline $\begin{array}{l}\text { Osteoid volume (bone tissue } \\
\text { referent) }\end{array}$ & $\%$ & $\mathrm{OV} / \mathrm{BV}$ & Percent of bone tissue occupied by osteoid $=100 *(\mathrm{O} . \mathrm{Ar} / \mathrm{B} \cdot \mathrm{Ar})$ \\
\hline Osteoid thickness & $\mu \mathrm{m}$ & O.Th & Average thickness of osteoid seams $=(\pi * \mathrm{O} . \mathrm{Wi}) /(4 * \mathrm{~N} . \mathrm{O} . \mathrm{Wi})$ \\
\hline Wall thickness & $\mu \mathrm{m}$ & W.Th & Average thickness of osteons $=(\pi * \mathrm{~W} . \mathrm{Wi}) /(4 * \mathrm{~N} . \mathrm{W} . \mathrm{Wi})$ \\
\hline Mineral apposition rate & $\mu \mathrm{m} / \mathrm{d}$ & MAR & Mineral apposition rate $=(\mathrm{iL} . \mathrm{Wi} * \pi) /(\mathrm{d} * 4) / \mathrm{N} . \mathrm{iL} . \mathrm{Wi}$ \\
\hline Adjusted mineral appositional rate & $\mu \mathrm{m} / \mathrm{d}$ & Aj.AR & $\begin{array}{l}\text { Mineral apposition rate adjusted for osteoid surface bearing } \\
\text { label=MAR*(MS/OS }) / 100\end{array}$ \\
\hline Osteoid surface & $\%$ & OS/BS & Percent of bone surface occupied by osteoid=100*O.Pm/B.Pm \\
\hline Osteoblast surface & $\%$ & Ob.S/BS & Percent of bone surface occupied by osteoblasts=100*Ob.Pm/B.Pm \\
\hline Double label surface & $\%$ & $\mathrm{dLS} / \mathrm{BS}$ & Percent of bone surface occupied by double label $=100 * \mathrm{dL} \cdot \mathrm{Pm} / \mathrm{B} . \mathrm{Pm}$ \\
\hline Single label surface & $\%$ & sLS/BS & Percent of bone surface occupied by single label=100*sL.Pm/B.Pm \\
\hline Mineralizing surface & $\%$ & MS/BS & $=100 *(\mathrm{dL} \cdot \mathrm{Pm}+0.5 \mathrm{sL} \cdot \mathrm{Pm}) / \mathrm{B} \cdot \mathrm{Pm}$ \\
\hline Eroded (resorptive) surface & $\%$ & ES/BS & Percent of bone surface occupied by resorption surface $=100 *($ E.Pm/B.Pm $)$ \\
\hline Osteoclast surface & $\%$ & Oc.S/BS & Percent of bone surface occupied by osteoclasts $=100 *(\mathrm{Oc} . \mathrm{Pm} / \mathrm{B} . \mathrm{Pm})$ \\
\hline Percentage of osteoid mineralizing & $\%$ & MS/OS & Percent of osteoid surface mineralizing $=100 *(\mathrm{MS} / \mathrm{BS}) /(\mathrm{OS} / \mathrm{BS})$ \\
\hline Mineralization lag time & $\mathrm{d}$ & Mlt & $\begin{array}{l}\text { Average time lag for mineralization of osteoid }=(\mathrm{O} . \mathrm{Th} * \mathrm{OS} / \mathrm{BS}) /(\mathrm{MAR} * \mathrm{MS} / \\
\text { BS) }\end{array}$ \\
\hline Osteoid maturation time & $\mathrm{d}$ & Omt & Time for mineralization of osteoid at forming sites $=\mathrm{O}$.Th/MAR \\
\hline $\begin{array}{l}\text { Bone formation rate (bone surface } \\
\text { ref.) }\end{array}$ & $\mu \mathrm{m}^{3} / \mu \mathrm{m}^{2} /$ year & $\mathrm{BFR} / \mathrm{BS}$ & Rate of bone formation, surface referent=MAR*MS/BS*365.25 \\
\hline $\begin{array}{l}\text { Bone formation rate (bone volume } \\
\text { ref.) }\end{array}$ & $\% /$ year & $\mathrm{BFR} / \mathrm{BV}$ & $\begin{array}{l}\text { Rate of bone formation (bone volume referent })=((\mathrm{BS} / \mathrm{TV}) *(\mathrm{MS} / \\
\mathrm{BS}) * \mathrm{MAR} * 365.25 * 100) /(\mathrm{BV} / \mathrm{TV}) * 0.01\end{array}$ \\
\hline Activation frequency & \#/year & Ac.f & $\begin{array}{l}\text { Frequency of appearance of new remodeling units at one location=(BFR/ } \\
\text { BS)/W.Th*0.001 }\end{array}$ \\
\hline Cortical thickness \#1 & $\mu \mathrm{m}$ & Ct1.Th & Thickness of Cortex\#1=Ct1.Wi/N.Ct1.Wi \\
\hline Cortical porosity \#1 & $\%$ & Ct1.Th & Porosity of Cortex\#1=100*Ct1.Po.Ar/Ct1.Ar \\
\hline Cortical thickness \#2 & $\mu \mathrm{m}$ & $\mathrm{Ct} 2 . \mathrm{Th}$ & Thickness of Cortex\#2=Ct2.Wi/N.Ct2.Wi \\
\hline Cortical porosity \#2 & $\%$ & $\mathrm{Ct} 2 . \mathrm{Th}$ & Porosity of Cortex $\# 2=100 * \mathrm{Ct} 2 . \mathrm{Po} . \mathrm{Ar} / \mathrm{Ct} 2 . \mathrm{Ar}$ \\
\hline
\end{tabular}


Table 3

Variables that may be reported.

\begin{tabular}{|c|c|c|c|}
\hline Variables & Units & Symbol & Definition/formula \\
\hline Surface density & $\mathrm{mm}^{2} / \mathrm{mm}^{3}$ & $\mathrm{BS} / \mathrm{TV}$ & Surface to volume ratio=Tt.Pm/Tt.Ar \\
\hline Osteoblast number & $\# / \mathrm{mm}$ & Ob.N/B.Pm & Number of osteoblasts seen per mm in standard sections \\
\hline Osteoclast number & $\# / \mathrm{mm}$ & Oc.N/B.Pm & Number of osteoclasts seen per $\mathrm{mm}$ in standard sections \\
\hline Trabecular thickness & $\mu$ & Tb.Th & Average thickness of trabeculae $=(\mathrm{BV} / \mathrm{TV}) * 20 /(\mathrm{BS} / \mathrm{TV})$ \\
\hline Trabecular number & $\# / \mathrm{mm}$ & Tb.N & Number of trabecular silhouettes $=(\mathrm{BV} / \mathrm{TV}) * 10 / \mathrm{Tb} . \mathrm{Th}$ \\
\hline Trabecular separation & $\mu$ & Tb.Sp & Average distance between trabeculae $=(1000 / \mathrm{TbN})-(\mathrm{Tb} . \mathrm{Th} / 10)$ \\
\hline Erosion depth & $\mu$ & E.D & Erosion depth=E.D \\
\hline Reversal surface & $\%$ & $\mathrm{RvS} / \mathrm{BS}$ & Percent trabecular bone in reversal phase $=(\mathrm{RvPm} / \mathrm{B} . \mathrm{Pm}) * 100$ \\
\hline Trabecular fibrosis surface & $\%$ & $\mathrm{Fb} . \mathrm{S} / \mathrm{BS}$ & Percent trabecular bone with fibrous surface $=(\mathrm{Fb} . \mathrm{Pm} / \mathrm{B} . \mathrm{Pm}) * 100$ \\
\hline Formation period & $\mathrm{d}$ & FP & Average osteon formation period=(W.Th*OS/BS $) /(\mathrm{MS} / \mathrm{BS} * \mathrm{MAR})$ \\
\hline Resorption period & $\mathrm{d}$ & Rs.P & Average osteon resorption period $=(\mathrm{FP} * \mathrm{ES} / \mathrm{BS}) / \mathrm{OS} / \mathrm{BS}$ \\
\hline Remodeling period & $\mathrm{d}$ & Rm.P & Average osteon remodeling period $=\mathrm{FP}+\mathrm{Rs} . \mathrm{P}$ \\
\hline $\begin{array}{l}\text { Bone formation Rate (total volume } \\
\text { ref.) }\end{array}$ & $\% /$ year & $\mathrm{BFR} / \mathrm{TV}$ & $\begin{array}{l}\text { Rate of bone formation, total volume referent }=(\mathrm{MAR} * 365.25 *(\mathrm{~T} . \mathrm{Pm} / \\
\text { Tt.Ar }) / 0.01\end{array}$ \\
\hline
\end{tabular}




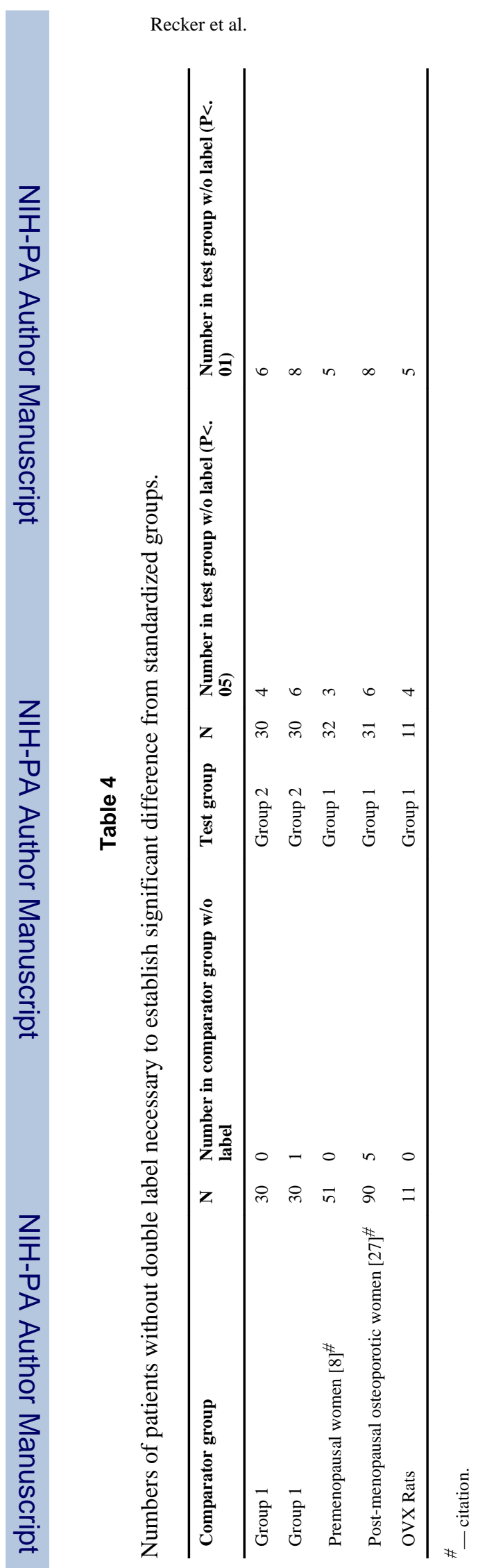

Page 21

Bone. Author manuscript; available in PMC 2012 November 1. 